\title{
Coexpression of Two Visual Pigments in a Photoreceptor Causes an Abnormally Broad Spectral Sensitivity in the Eye of the Butterfly Papilio xuthus
}

\author{
Kentaro Arikawa, ${ }^{1}$ Shin Mizuno, ${ }^{1}$ Michiyo Kinoshita, ${ }^{1}$ and Doekele G. Stavenga ${ }^{2}$ \\ ${ }^{1}$ Graduate School of Integrated Science, Yokohama City University, Yokohama 236-0027, Japan, and ${ }^{2}$ Department of Neurobiophysics, University of \\ Groningen, 9747 AG, Groningen, The Netherlands
}

The compound eye of the butterfly Papilio xuthus consists of three different types of ommatidia, each containing nine photoreceptor cells (R1-R9). We have found previously that the R5-R8 photoreceptors of type II ommatidia coexpress two different mRNAs, encoding opsins of green- and orange-red-absorbing visual pigments (Kitamoto et al., 1998). Do these cells contain two functionally distinct visual pigments? First, we identified the sensitivity spectrum of these photoreceptors by using combined intracellular recording and dye injection. We thus found that the R5-R8 of type II ommatidia have a characteristic sensitivity spectrum extending over an excessively broad spectral range, from the violet to the red region; the photoreceptors are therefore termed broadband photoreceptors. The spectral shape was interpreted with a computational model for type II ommatidia, containing a UV visual pigment in cells R1 and R2, two green visual pigments in cells R3 and R4, a far-UV-absorbing screening pigment (3-hydroxyretinol) in the distal part of the ommatidium, and a red-screening pigment that surrounds the rhabdom. The modeling suggests that both visual pigments in the R5-R8 photoreceptors participate in phototransduction. This work provides the first compelling evidence that multiple visual pigments participate in phototransduction in single invertebrate photoreceptors.

Key words: vision; color; optical filters; insects; rhodopsin; signal transduction

\section{Introduction}

It is a generally accepted concept in vision research that single photoreceptors contain a single type of visual pigment. However, recent studies have demonstrated that some photoreceptors violate this dogma: they coexpress multiple visual pigments (Roehlich et al., 1994; Sakamoto et al., 1996; Kitamoto et al., 1998; Glosmann and Ahnelt, 2002; Parry and Bowmaker, 2002). For example, a subset of murine cone photoreceptors contains both shortwave- and midwave-absorbing visual pigments (Applebury et al., 2000). An obvious question that arises is whether these visual pigments participate in phototransduction. Some electrophysiological measurements have been performed (Makino and Dodd, 1996; Lyubarsky et al., 1999), but such attempts are rather rare, and therefore additional proof would be desirable.

In the course of studies on the color vision of the butterfly Papilio xuthus, we discovered that some photoreceptors coexpress two mRNAs encoding different visual pigment opsins (Kitamoto et al., 1998). This report addresses whether these photoreceptors contain two physiologically active visual pigments.

An ommatidium of Papilio contains nine photoreceptors (R1-R9) (see Fig. 1a). The rhabdomeres of the photoreceptors, harboring the visual pigments, together form the fused rhabdom, a cylindrical structure that acts as an optical waveguide. Clusters

Received 0ct. 15, 2002; revised March 11, 2003; accepted March 13, 2003.

This work was supported by research grants from the Ministry of Education, Culture, Sports, Science and Technology and the Japan Science and Technology Corporation to K.A. Shin-ya Takemura participated in part of the electrophysiological experiments.

Correspondence should be addressed to Dr. Kentaro Arikawa, Graduate School of Integrated Science, Yokohama City University, 22-2 Seto, Kanazawa-ku, Yokohama 236-0027, Japan. E-mail: arikawa@yokohama-cu.ac.jp.

Copyright $\odot 2003$ Society for Neuroscience $\quad 0270-6474 / 03 / 234527-06 \$ 15.00 / 0$ of either red or yellow pigment, lining the rhabdom (see Fig. 1b) (perirhabdomeral pigmentation), act as filters of light propagating along the rhabdom. According to the pigmentation, Papilio ommatidia can be divided into three types: type I are strongly red pigmented and nonfluorescent, type II are pale-red pigmented and emit a whitish fluorescence under UV light (see Fig. 1c), and type III are yellow pigmented and nonfluorescent (Arikawa and Stavenga, 1997; see cover of this issue).

We found five classes of spectral receptors in the Papilio retina, peaking in the UV, violet, blue, green, and red wavelength regions (Arikawa et al., 1987). We localized the receptors in the three types of ommatidia by in situ hybridization (see Fig. 1, table): we found that the Papilio retina expresses five mRNAs of visual pigment opsins, termed P. xuthus $\mathrm{UV}$ (PxUV), blue (PxB), and long-wavelength 1-3 (PxL1-PxL3) (Kitamoto et al., 1998, 2000), creating UV-, blue-, and long-wavelength-absorbing visual pigments, respectively. By combining this finding with the electrophysiological results, we concluded that PxL1 and PxL2 construct green-absorbing visual pigments, whereas PxL3 constructs orange-red-absorbing visual pigment. Interestingly, we found a subset of ommatidia whose R5-R8 photoreceptors were positive for both PxL2 and PxL3 mRNAs (see Fig. 1d,e; highlighted in the table). If both visual pigments trigger the phototransduction process in a photoreceptor cell, the sensitivity spectrum should be broader than those of receptors containing only PxL2 or PxL3.

In our early recordings, we had encountered units with broad sensitivity spectra. We initially considered that they might originate from multiple photoreceptors, artificially coupled because of low-quality penetrations. However, detailed analysis of the 
units, combining electrophysiology, histology, and computational modeling, provided evidence that the recordings were in fact from single R5-R8 photoreceptors of type II ommatidia. In this report, we present the results that led to this conclusion, and we will argue that both PxL2 and PxL3 are physiologically active in these photoreceptors.

\section{Materials and Methods}

Animals. Spring-form males of the Japanese yellow swallowtail butterfly $P$. xuthus were used within $3 \mathrm{~d}$ of emergence. The butterflies were reared on fresh citrus leaves at $25^{\circ} \mathrm{C}$ under an $8 / 16 \mathrm{hr}$ light/dark cycle. The pupae were stored at $4^{\circ} \mathrm{C}$ for at least 3 months and then allowed to emerge at $25^{\circ} \mathrm{C}$.

We restricted the following analysis to the ventral two-thirds of the retina, because in the dorsal one-third, the cornea fluoresces under UV illumination, which obscures the ommatidial fluorescence in type II ommatidia. Type II ommatidia are presumably absent in the dorsal region (Arikawa and Stavenga, 1997).

Electrophysiology. A butterfly whose wings and legs were removed was fixed on a plastic stage with its dorsal side up and then mounted in the electrophysiological setup in a Faraday cage. A silver wire inserted in the stump of an antenna served as the indifferent electrode. To insert a glass micropipette into the eye, a hole covering $\sim 10-20$ facets was made in the dorsal region of the eye with a razor blade.

Monochromatic light stimuli were provided by a $500 \mathrm{~W}$ xenon arc lamp through 22 narrow-band interference filters ranging from 300 to $700 \mathrm{~nm}$. The intensity of the light stimulus was attenuated with neutraldensity filters and an optical wedge with a range of 4 log units. The light was focused on the tip of an optical fiber that led the light into the cage. The other end of the optical fiber was attached to a Cardan arm perimeter device, where it provided a point source of light ( $1.6^{\circ}$ in diameter). The quantum flux of each monochromatic stimulus was measured by a radiometer (model 470D; Sanso, Tokyo, Japan), and the maximum quantum flux of each monochromatic stimulus at the corneal surface was adjusted with another optical wedge to $5.0 \times 10^{11}$ photons $\cdot \mathrm{cm}^{-2} \cdot \mathrm{sec}^{-1}$ $\left(\sim 2.5 \times 10^{6}\right.$ photons $\cdot$ facet $^{-1} \cdot \mathrm{sec}^{-1}$, with a facet diameter of $\left.25 \mu \mathrm{m}\right)$.

A glass micropipette filled with $5 \%$ Lucifer yellow aqueous solution (resistance, $\sim 150 \mathrm{M} \Omega$ ) was inserted into the retina through the hole made in the cornea. A successful penetration of a photoreceptor cell was deduced from a resting potential of approximately $-50 \mathrm{mV}$ and responsivity to dim light. The optical axis of the responding unit was located by moving the tip of the optical fiber until dim white light flashes produced a maximal response.

We recorded from nearly dark-adapted photoreceptors. To minimize the effect of light adaptation during the experiment, we used light flashes that were limited to $30 \mathrm{msec}$ in duration. This is the shortest light stimulus that still produces a receptor potential with an amplitude of the initial transient component that is the same as that elicited by longer (e.g., $1 \mathrm{sec})$ stimuli.

The receptor potentials were recorded through a preamplifier (MEZ7200; Nihon-kohden, Tokyo, Japan) connected to an oscilloscope (VC11; Nihon-kohden). First, the spectral type of the unit was determined by a series of monochromatic flashes with an intensity of $5.0 \times 10^{9} \sim 10^{10}$ photons $\cdot \mathrm{cm}^{-2} \cdot \mathrm{sec}^{-1}$. When the spectral response profile indicated a broadband unit, we measured the response-stimulus intensity $(V-\log I)$ functions at several wavelengths in the intensity range of $5.0 \times 10^{7} \sim 10^{11}$ photons $\cdot \mathrm{cm}^{-2} \cdot \mathrm{sec}^{-1}\left(\sim 2.5 \times 10^{2} \sim 10^{6}\right.$ photons $\left.\cdot \mathrm{facet}^{-1} \cdot \mathrm{sec}^{-1}\right)$ in some initial cases. Data were fitted to the Naka-Rushton function: $V / V_{\max }=$ $I^{n} /\left(I^{n}+K^{n}\right)$, where $I$ is the stimulus intensity, $V$ is the response amplitude, $V_{\max }$ is the maximum response amplitude, $K$ is the stimulus intensity eliciting $50 \% V_{\max }$, and $n$ is the exponent. We thus confirmed that the $V_{\max }$ and $n$ are not significantly different between $V$ - $\log I$ functions recorded at different wavelengths. We therefore recorded a single $V-\log I$ function at $540 \mathrm{~nm}$ for each unit. The best fit of the Naka-Rushton function was taken as the $V-\log I$ function of the given unit.

Recorded units were subjected to additional analysis only when the $V_{\max }$ was $>40 \mathrm{mV}$. Amplitudes of the spectral responses were extrapolated to the $V-\log I$ function of a given unit to transform the amplitudes into photon numbers required for the responses. The normalized reciprocal of the relative photon numbers then yielded the sensitivity.

The recorded broadband units were marked by Lucifer yellow. We delivered Lucifer yellow from the tip of the electrode by applying a hyperpolarizing DC of 2-5 nA for 5-10 min. The animal was then unmounted from the Faraday cage and positioned under an epifluorescence microscope (BX-60; Olympus Optical, Tokyo, Japan). The ommatidium containing the Lucifer yellow-injected unit was identified and photographed under violet excitation (dichroic cube U-MNBV; excitation bandpass filter at $420 \mathrm{~nm}$ and emission cutoff filter at $470 \mathrm{~nm}$ ). Subsequently, the excitation light was switched to UV (dichroic cube U-MWU; excitation bandpass filter at $350 \mathrm{~nm}$ and emission cutoff at $420 \mathrm{~nm}$ ) to correlate the stained ommatidium with the array of fluorescing ommatidia.

Histology. After the electrophysiology and the fluorescence microscopy, the compound eye containing the Lucifer yellow-injected unit was processed for light-microscopic histology. The eye was isolated and fixed at room temperature for $30 \mathrm{~min}$ in $4 \%$ paraformaldehyde in $0.1 \mathrm{M}$ sodium cacodylate buffer at $\mathrm{pH} 7.4$ and then dehydrated with an acetone series and embedded in Spurr's resin. Sections (10-14 $\mu \mathrm{m}$ thick) were cut with a rotary microtome. The sections were first observed under the epifluorescence microscope with violet excitation to localize the unit within the ommatidium, and subsequently normal transmission light was used to identify the color of the ommatidial pigmentation.

Modeling. The computational model used for calculating the sensitivity spectrum of the R5-R8 cells (see Figs. 2, 4) was essentially identical to that of Arikawa et al. (1999a,b), but the parameters used were slightly adjusted. We calculated the absorption of light entering type II ommatidia on-axis in the individual photoreceptors (R1-R9). The change in light flux along the rhabdom was described by Snyder et al. (1973) as follows:

$$
\begin{aligned}
(d I(z, \lambda) / d z)=-\left\{\eta(\lambda) \sum_{j=1}^{9} f_{j}(z) \kappa_{j}(z) \alpha_{j}(\lambda)\right. & \\
& {\left.[1-\eta(\lambda)] \kappa_{s}(z) \alpha_{s}(\lambda)\right\} I(z, \lambda), }
\end{aligned}
$$

where $I(z, \lambda)$ is the light flux at a distance $z$ from the tip of the rhabdom, $\eta(\lambda)$ is the fraction of the light flux propagated within the rhabdom boundary, $\lambda$ is the wavelength of light, $f_{j}$ is the fractional cross-section taken up by photoreceptor $\mathrm{R}_{j}(j=1-9), \kappa_{j}$ is the peak absorbance coefficient of visual pigment $j, \kappa_{s}$ is the peak absorbance coefficient of the red-screening pigment in the photoreceptor, and $\alpha_{j}$ and $\alpha_{s}$ are the (normalized) spectral absorption coefficients of the visual and screening pigments, respectively. The light absorbed by the visual pigment in each photoreceptor, integrated over the length of the photoreceptor, yields its absorption spectrum. Normalization then yields the sensitivity spectrum.

We have modeled the rhabdom as a circular cylinder. On the basis of detailed anatomy, the assumptions were as follows. The total length of the rhabdom is $500 \mu \mathrm{m}$; a distal layer of $260 \mu \mathrm{m}$ is fully occupied by the R1-R4 photoreceptors; in a transition zone of $70 \mu \mathrm{m}$, the R1-R4 rhabdomeres reduce in size, whereas the participation of the R5-R8 cells increases; the latter cells fully occupy the proximal $140 \mu \mathrm{m}$; the basal 30 $\mu \mathrm{m}$ is taken up by the R9 photoreceptor; and each of the R1-R4 and R5-R8 occupy one-quarter of the rhabdomere cross-sections in the distal and proximal layers, respectively. The fluorescing, UV-absorbing 3-hydroxyretinol is homogeneously distributed in the distal $70 \mu \mathrm{m}$, with an absorption coefficient of $0.2 \mu \mathrm{m}^{-1}$. The red-screening pigment is present in cells R3-R8, with an absorption coefficient of $0.005 \mu \mathrm{m}^{-1}$. The visual pigment peak wavelengths are $365 \mathrm{~nm}$ (PxUV), $530 \mathrm{~nm}$ (PxL1), $515 \mathrm{~nm}$ (PxL2), and $575 \mathrm{~nm}$ (PxL3); the photoreceptors R1 and R2 both contain exclusively PxUV; R3 and R4 contain equal amounts of PxL1 and PxL2; R5-R8 contain PxL2 and PxL3 in a concentration ratio of 3:1 (see below); and R9 contains both PxL1 and PxL2. The visual pigment spectra are described by template formulas (Stavenga et al., 1993). Contribution of metarhodopsin was neglected here, because the photoreceptors were nearly dark-adapted in the present experimental condition. The photoreceptors R3-R9 are polarization sensitive, with a polarization 
a

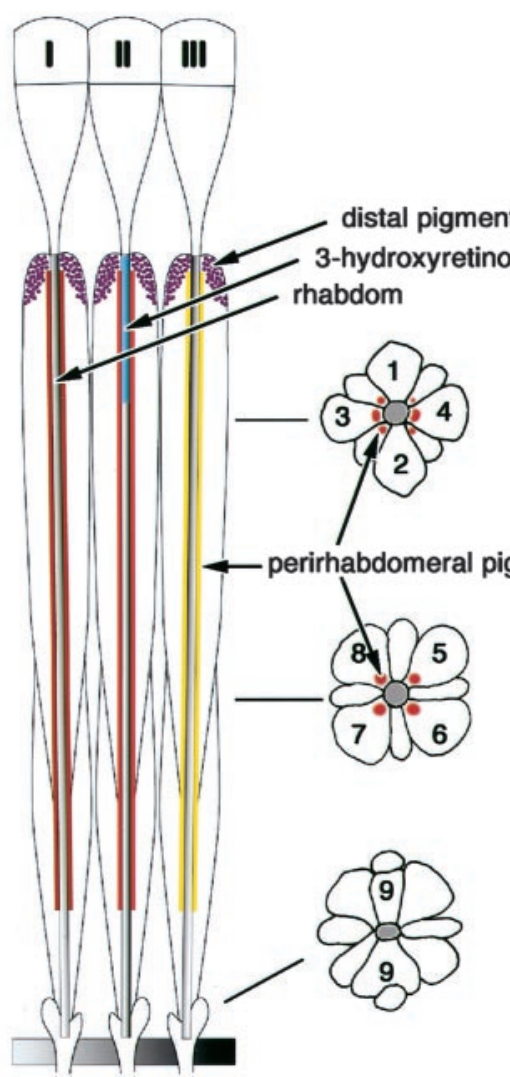

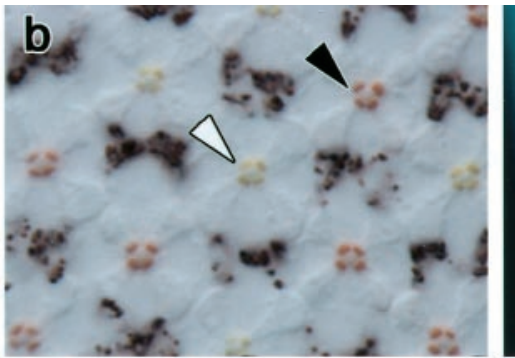
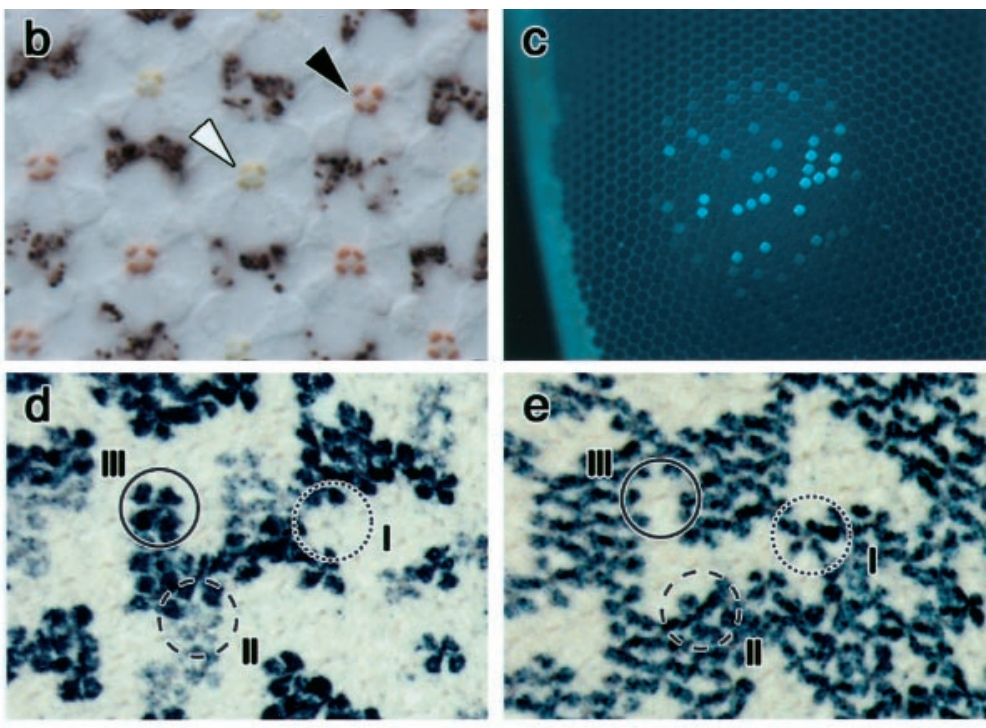

\begin{tabular}{|c|c|c|c|c|c|c|}
\hline \multirow{2}{*}{$\begin{array}{r}\text { Type of ommatidia: } \\
\text { Pigmentation: } \\
\text { Fluorescence: } \\
\text { Photoreceptors }\end{array}$} & \multicolumn{2}{|c|}{$\begin{array}{c}\text { Type I } \\
\text { red } \\
\text { No } \\
\end{array}$} & \multicolumn{2}{|c|}{$\begin{array}{c}\text { Type II } \\
\text { red } \\
\text { Yes }\end{array}$} & \multicolumn{2}{|c|}{$\begin{array}{c}\text { Type III } \\
\text { yellow } \\
\text { No }\end{array}$} \\
\hline & $S(\lambda)$ & opsin & $S(\lambda)$ & opsin & $S(\lambda)$ & opsin \\
\hline R1 & UV* & PxUV* & V & PxuV & B & PxB \\
\hline R2 & $\mathrm{B}^{*}$ & PxB' & V & PxUV & B & PxB \\
\hline R3-4 & $D G$ & PxL1, PxL2 & $S G$ & PxL1, PxL2 & $D G$ & PxL1, PxL2 \\
\hline R5-8 & $R$ & PXL3 & BB & PxL2, PxL3 & $D G$ & PxL2 \\
\hline R9 & $?$ & PxL1, PxL2 & $?$ & PxL1, PxL2 & $?$ & PXL1, PxL2 \\
\hline
\end{tabular}

Figure 1. Anatomy of the compound eye of P. xuthus; the dorsal side corresponds to the top edge of the micrographs. a, Diagram of three types of ommatidia (from left to right, types I-III). Each ommatidium contains nine photoreceptors, R1-R9 (1-9). R1-R4 are distal photoreceptors, which form the distal two-thirds of the rhabdom; R5-R8 are proximal photoreceptors, forming the proximal one-third of the rhabdom; and R9 is a basal cell. R1 and R2 contain distally purple pigment granules (distal pigment). Types I and II have red pigmentation around the rhabdom, whereas type III has yellow pigmentation (perirhabdomeral pigment). In type Il, a fluorescing pigment, 3-hydroxyretinol, is concentrated in the distal portion of the photoreceptor layer (indicated by blue). $b$, Transverse, unstained section through the proximal tier of the retina, showing red (black arrowhead; type I or II ommatidium) or yellow (white arrowhead; type III ommatidium) pigmentation around the rhabdom. c, UV-induced fluorescence caused by 3-hydroxyretinol marks the type II ommatidia (from eye in intact, living animal). d, e, In situ hybridization of PxL2 (green-absorbing visual pigment) $(d)$ and PxL3 (orange-red-absorbing visual pigment) (e) mRNA in adjacent sections through the proximal tier. R5-R8 are exclusively labeled with either the PxL2 probes (solid circles; type III) or the PxL3 probe (dotted circles; type I). R5-R8 of type II ommatidia are labeled by both PxL2 and PxL3 probes (dashed circles). The table is a summary of the characteristics of the three ommatidial types. In addition to the perirhabdomeral pigmentation and the autofluorescence, it presents the spectral sensitivity of the photoreceptors $[S(\lambda)]$ and the visual pigment opsins expressed in the ventral half of the eye. $V$, Violet; $B$, blue; $D G$, double-peaked green; $S G$, single-peaked green; $\mathrm{R}$, red; $\mathrm{BB}$, broadband. The sensitivity spectrum of $\mathrm{R} 9$ photoreceptors is an open question; it probably peaks in the orange wavelength range, but electrophysiological data are lacking so far. For details of $S(\lambda)$, see Bandai et al. (1992), and for visual pigments, see Kitamoto et al. (1998, 2000). For type I, the spectral type of R1 and R2 may be interchanged (asterisks).

sensitivity (PS) of 2, whereas for R1 and R2, whose microvilli curve in two directions (Arikawa et al., 1999a), PS = 1; or the dichroic ratio of the UV visual pigment and that of the other visual pigments are 1 and 2, respectively. The direction of the polarization peak sensitivity with respect to the vertical axis of the body is $90^{\circ}$ for cells $\mathrm{R} 3$ and $\mathrm{R} 4,35^{\circ}$ for $\mathrm{R} 5$ and $\mathrm{R} 7$, and $125^{\circ}$ for R6 and R8 (Bandai et al., 1992). The total (maximal) absorption coefficient of the rhabdomeric tissue of all photoreceptors is $\kappa_{j}$ $=0.005 \mu \mathrm{m}^{-1}$ for $j=1-9$ (Warrant and Nilsson, 1998). The rhabdomere diameter is $2.6 \mu \mathrm{m}$. The first waveguide mode was assumed to be the dominant component of the light flux propagated in the rhabdom, because the wavelength dependence of the second-order mode is very similar to that of the first mode, and the relative contributions of the modes to the spectral sensitivity have not yet been established. The fraction of light within the rhabdom $[\eta(\lambda)]$ was calculated using the waveguide optics formulas for insect photoreceptors (Snyder et al., 1973; Stavenga, 2003).

A relative amount of PxL2 and PxL3 of 3:1 was chosen, because this ratio gave satisfactory outcomes in the computer simulations. We have noticed that the in situ hybridization results did not necessarily match this choice: the labeling density of PxL2 is comparable with or maybe even weaker than that of PxL3 (Fig. 1d,e). However, taking equal concentrations of PxL2 and PxL3 yielded a much too high sensitivity in the long-wavelength range in the simulations. This may imply that the label- ing density of in situ hybridization does not provide a direct measure of the expressed visual pigment concentration in photoreceptors. Another possible cause of the discrepancy is the limited accuracy of the predicted absorption peak wavelength of the PxL2 and PxL3 visual pigments. We predicted absorption peaks of $515 \mathrm{~nm}$ for PxL2 and $575 \mathrm{~nm}$ for PxL3 only by model calculation (Arikawa et al., 1999a), not by direct measurements. Direct proof of the absorption spectra requires expression experiments (Feiler et al., 1992; Shaaban et al., 1998; Townson et al., 1998; Jacobs et al., 1999).

\section{Results}

The proximal photoreceptors of the fluorescent type II ommatidia express a broadband spectral sensitivity

In the course of previous studies on the photoreceptors of $P$. xuthus, we sometimes encountered units with abnormally broad sensitivity spectra, but we could not unequivocally prove that the recordings were from single photoreceptors. We therefore designed the experimental procedure described below.

With the electrode advancing in the retina, we searched for units with broad sensitivity spectra. Broadband units were detected only when the electrode track ran through the proximal tier of the ventral retina. Figure $2 a$ shows a typical recording 
trace, which contains two series of spectral responses $(300-700 \mathrm{~nm}$; with equiquantal monochromatic flashes) and $V-\log I$ functions (recorded at 560 and $440 \mathrm{~nm}$ in this case). Figure $2 b$ is a set of $V-\log I$ functions recorded from a single broadband unit at 420, 440, 540, and $620 \mathrm{~nm}$, with the best fits of the Naka-Rushton function. The maximum response amplitude, $V_{\max }$, and the exponent, $n$, are virtually identical for the different recording wavelengths (for values, see Fig. 2 legend). The sensitivity spectrum presented in Figure $2 c$ shows the average of 10 cells from nine butterflies.

We identified and localized all of the recorded units histologically, as follows, to determine whether the units correspond to single photoreceptors. After the electrophysiological recordings, we delivered Lucifer yellow from the electrode tip by applying a hyperpolarizing DC. The minor amount of Lucifer yellow that may have leaked into the recorded photoreceptor during the spectral measurements before applying the current did not perturb the sensitivity spectra, because the spectra of known spectral receptors encountered in the course of the present study were identical to those of our previous recordings with $3 \mathrm{M} \mathrm{KCl}$-filled electrodes (Arikawa et al., 1987; Bandai et al., 1992).

The ommatidium containing the Lucifer yellow-injected unit was subsequently localized by fluorescence microscopy, applying violet epi-illumination (Fig. $3 a$ ). By switching the excitation light to UV, we found that the ommatidium emitted whitish fluorescence, proving that the unit was a member of a type II ommatidium (Fig. $3 b)$. The eyes were then further processed for histology to identify the receptor type. In the particular case of Figure $3 c$, showing a transverse section through the proximal tier of the ommatidia, the unit appeared to be a single R8 photoreceptor. Regular transmission microscopy of the same frame revealed that the Lucifer yellow-injected photoreceptor contained red pigment (Fig. $3 d$ ), in agreement with the previous observation that type II ommatidia are red pigmented (Arikawa and Stavenga, 1997).

Identical observations were made for all of the broadbandsensitive units analyzed in the present study (i.e., the broadband units were invariably identified as belonging to the set of proximal photoreceptors, R5-R8, of the fluorescent type II ommatidia).

\section{Modeling of the broadband sensitivity spectrum}

In modeling the broadband sensitivity spectra, we have calculated the fraction of light entering the rhabdom of type II ommatidia that is absorbed by the visual pigments as well as by the screening pigments to be in the wavelength range of 300-700 $\mathrm{nm}$. We have assumed that both PxL2 and PxL3 visual pigments in R5-R8 photoreceptors contribute to the spectral sensitivity in an identical manner (i.e., each absorbed photon by one or the other visual pigment contributes equally to phototransduction or opens the same number of channels). Figure $2 c$ shows the results of the model calculations of the normalized absorption spectra of the $\mathrm{R} 5-\mathrm{R} 8$ receptors, together with the average of the broadband sensitivity spectra. We calculated the spectra for incident light with different polarization angles (i.e., $0,90,35$, and $125^{\circ}$, respectively). The calculated and measured spectra closely match at the short and the long wavelengths, but the spectra vary in the wavelength range of 480-580 $\mathrm{nm}$. The variation can be attributed primarily to the polarization sensitivity of the distal photoreceptors, R1-R4, which act as a polarization-dependent absorption filter for the proximal photoreceptors, R5-R8. For example, the $\mathrm{R} 3$ and R4 cells are single-peaked green receptors, peaking at 520 $\mathrm{nm}$ with maximum polarization sensitivity at $90^{\circ}$. When the ommatidium is stimulated with $90^{\circ}$ polarized light, the light is strongly absorbed by R3 and R4, thus reducing the light reaching the proximal tier and lowering the response of proximal photoreceptors in the middle wavelength region.

Figure 4 presents the calculated and recorded spectra of the proximal as well as distal photoreceptors in type II ommatidia. The R1 and R2 photoreceptors, which contain PxUV, a visual 

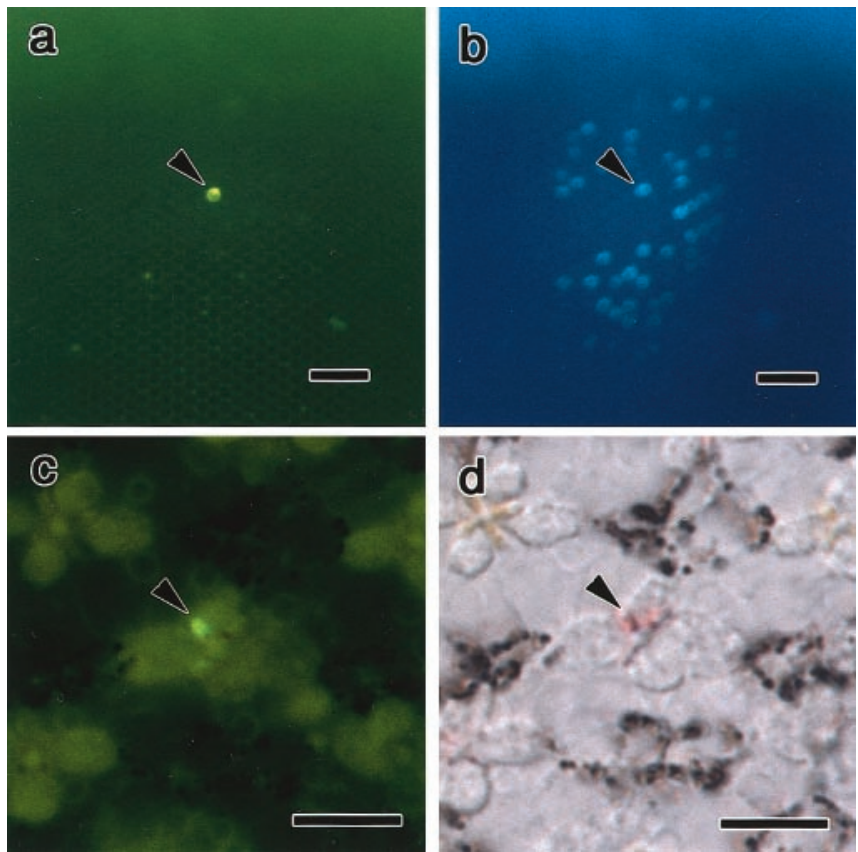

Figure 3. Light microscopic identification of the location of a broadband unit. The dorsal side of the eye, which was identified by the hole made in the cornea for electrode insertion (data not shown), corresponds to the top edge of all micrographs. The direction allows us to identify the photoreceptor number unambiguously (Fig. 1a). $a$, The ommatidium containing the Lucifer yellow-injected unit (arrowhead). b, UV excitation showing that the ommatidium was of type II (arrowhead). c, Section of the eye observed under violet excitation. The unit was a proximal photoreceptor R8 (arrowhead). $d$, Regular transmission microscopy revealed that the ommatidium of the labeled photoreceptor (arrowhead) contained red pigment. Scale bars: $a, b, 100$ $\mu \mathrm{m} ; c, d, 10 \mu \mathrm{m}$.

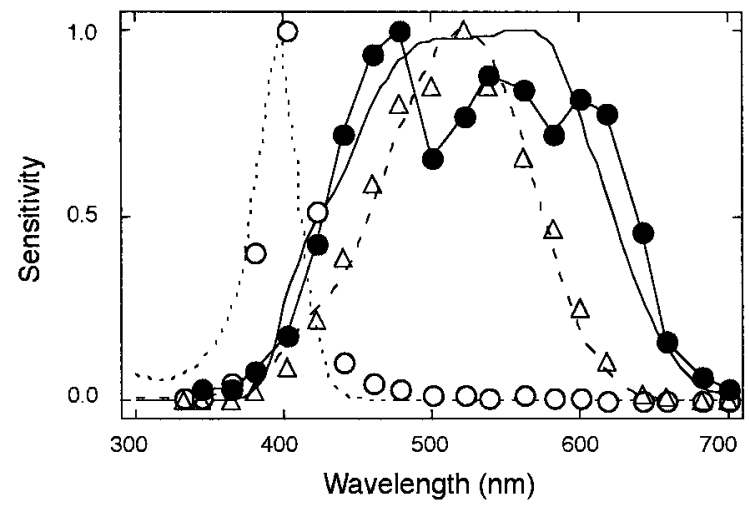

Figure 4. Calculated (R1 and R2, dotted curve; R3 and R4, dashed curve; R5-R8 at polarization $0^{\circ}$, solid curve) and recorded (violet receptor, open circles; single-peaked green receptor triangles; broadband receptor, filled circles) sensitivity spectra of the photoreceptor classes in type II ommatidia.

pigment absorbing maximally at $360 \mathrm{~nm}$, become violet receptors, with sensitivity spectrum peaking at $400 \mathrm{~nm}$, because of the abundant 3-hydroxyretinol concentrated in the distal part, which acts as a UV filter for the underlying photoreceptors (Arikawa et al., 1999b). The R3 and R4 photoreceptors, which contain PxL1 and PxL2, green visual pigments absorbing maximally at 530 and $515 \mathrm{~nm}$, respectively, become single-peaked green receptors, because they lose the usual $\beta$-band as a result of the UV filter, confirming our previous study on the type II ommatidium (Arikawa et al., 1999b). Similarly, the R5-R8 have negligible sensitivity in the ultraviolet because of the strongly UV-absorbing 3-hydroxyretinol. The R5-R8 contain PxL2 and PxL3, visual pigments absorbing maximally at 515 and $575 \mathrm{~nm}$, respectively, which are together responsible for the broadband spectral sensitivity, assuming that both visual pigments trigger phototransduction.

One might argue that the fluorescence of 3-hydroxyretinol could excite the photoreceptors in type II ommatidia. However, the recordings clearly indicate that this is not the case: the photoreceptors in type II ommatidia have virtually no sensitivity in the UV wavelength region (Fig. 4) in which the absorption of 3-hydroxyretinol exists. Although the fluorescence seems very prominent in micrographs (Fig. 1c), it requires extremely bright illumination provided by a focused mercury lamp of an epifluorescence microscope. Because of the low quantum yield, fluorescence will therefore have a negligible contribution to photoreceptor excitation.

\section{Discussion}

In this study, we describe a novel type of photoreceptor featured in the Papilio retina with an abnormally broad sensitivity spectrum, accordingly termed broadband receptor. Anatomically, the broadband-sensitive cells are the proximal photoreceptors (R5R8) in the type II ommatidia [i.e., the red-pigmented ommatidia that fluoresce under UV excitation (Figs. 1, 3)]. The photoreceptors were shown to express two mRNAs, encoding the visual pigment opsins PxL2 and PxL3 (Fig. 1d,e). The absorption characteristics of the corresponding visual pigments are spectrally quite different: the absorption peak wavelengths are in the green $(515 \mathrm{~nm})$ and orange-red $(575 \mathrm{~nm})$ regions, respectively (Arikawa et al., 1999a).

In the modeling, we incorporated the light-filtering effects of a number of optical absorbers, namely 3-hydroxyretinol in the distal part of the type II ommatidia, the red-screening pigment surrounding the rhabdom, and the visual pigments in the distal photoreceptors R1-R4, of which the visual pigments in the R3 and R4 photoreceptors filter specifically in the green wavelength region. The calculated absorption spectra reasonably fit to the broadband sensitivity spectra in the short- and the longwavelength regions. However, the modeling does not yet satisfactorily reproduce the bumpy profile of the sensitivity spectrum in the wavelength region between 480 and $580 \mathrm{~nm}$ (Fig. 2c). As described in Materials and Methods, we calculated the sensitivity spectrum by taking only the first-order waveguide mode into account. The second mode presumably contributes to the bumpy profile of the spectrum (Stavenga, 2003). To incorporate higherorder modes and additional details of spectral filtering, more detailed anatomy of the rhabdom geometry and fine structure will be necessary. Of course, some of the discrepancy of the model calculations and the recorded spectra may be caused by experimental errors. For instance, the alignment of the polarization angle of the stimulus light and the ommatidial dorsoventral axis is uncertain because of the slight distortion in the ommatidial lattice arrangement [Arikawa and Uchiyama (1996), their Fig. $3 c$. Nevertheless, the general correspondence of the measured broadband sensitivity spectrum with the computational results strongly suggests that both visual pigments contribute to phototransduction.

To the best of our knowledge, the broadband receptor of $P$. xuthus is the first clear example of a class of invertebrate photoreceptors with multiple visual pigments functioning simultaneously. It extends the as-yet-limited list of similar vertebrate photoreceptors (Makino and Dodd, 1996; Lyubarsky et al., 1999). The fact that the $V_{\max }$ value and $n$ value remain virtually constant between different stimulus wavelengths suggests that 
the response kinetics is wavelength independent (Fig. 2a,b). Therefore, it is likely that the two distinct visual pigments trigger the same transduction pathway. Of course, it is still possible that there are two independent transduction pathways, triggered by each visual pigment, whose kinetics is very similar.

The biological function of the broadband receptor for butterfly vision must remain a matter of speculation. It seems unlikely that it plays a role in color vision, a function that is logically conferred to the R5-R8 photoreceptors of the type I ommatidia: they have a narrow-band sensitivity spectrum, distinctly peaking in the red (Arikawa and Uchiyama, 1996; Arikawa et al., 1999a). Also, the R5-R8 photoreceptors of the type III ommatidia may well participate in color vision, because they have a clear spectrum peaking in the green. The broadband R5-R8 photoreceptors of type II ommatidia rather could play a role as general light intensity (or luminosity) detectors. Another possible function of broadband photoreceptors is related to the function of type II ommatidia themselves. The photoreceptors in type II ommatidia are virtually insensitive to UV because of the filtering effect of 3-hydroxyretinol [(Arikawa et al., 1999b), their Fig. 5]. Together with type I ommatidia, containing UV receptors (Fig. 1, table), the UV-insensitive type II ommatidia probably participate to enhance UV contrasts in the visual scene. Detecting UV contrasts is particularly important for flower visitors, because many flowers provide information on nectar localization by so-called nectar guides, which involve UV reflection/absorption (Heiling et al., 2003). The broadband photoreceptors are sensitive over the entire spectral range of butterfly vision, except in the UV range. Slight changes in UV intensity can therefore be efficiently analyzed by a combination of the broadband and UV receptors.

Butterfly eyes have a strikingly heterogeneous organization, with specific sets of spectral detectors combined in different types of ommatidia. Although the overall lattice of ommatidia is of crystalline regularity, the different types are randomly arranged within that regular lattice (Arikawa and Stavenga, 1997). Our working hypothesis is that the regular ommatidial lattice is connected to spatial vision and that the heterogeneous organization is related to color processing. Additional study is required to resolve in which way and to what extent the signals of the different photoreceptors feed into either of both visual modes.

\section{References}

Applebury ML, Antoch MP, Baxter LC, Chun LL, Falk JD, Farhangfar F, Kage K, Krzystolik MG, Lyass LA, Robbins JT (2000) The murine cone photoreceptor: a single cone type expresses both $\mathrm{S}$ and $\mathrm{M}$ opsins with retinal spatial patterning. Neuron 27:513-523.

Arikawa K, Stavenga DG (1997) Random array of colour filters in the eyes of butterflies. J Exp Biol 200:2501-2506.

Arikawa K, Uchiyama H (1996) Red receptors dominate the proximal tier of the retina in the butterfly Papilio xuthus. J Comp Physiol [A] 178:55-61.

Arikawa K, Inokuma K, Eguchi E (1987) Pentachromatic visual system in a butterfly. Naturwissenschaften 74:297-298.

Arikawa K, Scholten DGW, Kinoshita M, Stavenga DG (1999a) Tuning of photoreceptor spectral sensitivities by red and yellow pigments in the butterfly Papilio xuthus. Zool Sci 16:17-24.

Arikawa K, Mizuno S, Scholten DGW, Kinoshita M, Seki T, Kitamoto J, Stavenga DG (1999b) An ultraviolet absorbing pigment causes a narrow-band violet receptor and a single-peaked green receptor in the eye of the butterfly Papilio. Vision Res 39:1-8.

Bandai K, Arikawa K, Eguchi E (1992) Localization of spectral receptors in the ommatidium of butterfly compound eye determined by polarization sensitivity. J Comp Physiol [A] 171:289-297.

Feiler R, Bjornson R, Kirschfeld K, Mismer D, Rubin GM, Smith DP, Socolich M, Zuker CS (1992) Ectopic expression of ultraviolet-rhodopsins in the blue photoreceptor cells of Drosophila: visual physiology and photochemistry of transgenic animals. J Neurosci 12:3862-3868.

Glosmann M, Ahnelt PK (2002) A mouse-like retinal cone phenotype in the Syrian hamster: $\mathrm{S}$ opsin coexpressed with $\mathrm{M}$ opsin in a common cone photoreceptor. Brain Res 929:139-146.

Heiling AM, Herberstein ME, Chittka L (2003) Crab-spiders manipulate flower signals. Nature 421:334.

Jacobs GH, Fenwick JC, Calderone JB, Deeb SS (1999) Human cone pigment expressed in transgenic mice yields altered vision. J Neurosci 19:3258-3265.

Kitamoto J, Sakamoto K, Ozaki K, Mishina Y, Arikawa K (1998) Two visual pigments in a single photoreceptor cell: identification and histological localization of three mRNAs encoding visual pigment opsins in the retina of the butterfly Papilio xuthus. J Exp Biol 201:1255-1261.

Kitamoto J, Ozaki K, Arikawa K (2000) Ultraviolet and violet receptors express identical mRNA encoding an ultraviolet-absorbing opsin: identification and histological localization of two mRNAs encoding shortwavelength-absorbing opsins in the retina of the butterfly Papilio xuthus. J Exp Biol 203:2887-2894.

Lyubarsky AL, Falsini B, Pennesi ME, Valentini P, Pugh ENJ (1999) UVand midwave-sensitive cone-driven retinal responses of the mouse: a possible phenotype for coexpression of cone photopigments. J Neurosci 19:442-455.

Makino CL, Dodd RL (1996) Multiple visual pigments in a photoreceptor of the salamander retina. J Gen Physiol 108:27-34.

Parry JW, Bowmaker JK (2002) Visual pigment coexpression in guinea pig cones: a microspectrophotometric study. Invest Ophthalmol Vis Sci 43:1662-1665.

Roehlich P, Vanveen T, Szel A (1994) Two different visual pigments in one retinal cone cell. Neuron 13:1159-1166.

Sakamoto K, Hisatomi O, Tokunaga F, Eguchi E (1996) Two opsins from the compound eye of the crab Hemigrapsus sanguineus. J Exp Biol 199:441-450.

Shaaban SA, Corognale MA, Calderone JB, Huang J, Jacobs GH, Deeb SS (1998) Transgenic mice expressing a functional human photopigment. Invest Ophthalmol Vis Sci 39:1036.

Snyder AW, Menzel R, Laughlin SB (1973) Structure and function of the fused rhabdom. J Comp Physiol 87:99-135.

Stavenga DG (2003) Angular and spectral sensitivity of fly photoreceptors. I. Integrated facet lens and rhabdomere optics. J Comp Physiol [A] 189:1-17.

Stavenga DG, Smits RP, Hoenders BJ (1993) Simple exponential functions describing the absorbance bands of visual pigment spectra. Vision Res 33:1011-1017.

Townson SM, Chang BSW, Salcedo E, Chadwell LV, Pierce NE, Britt SG (1998) Honeybee blue-and ultraviolet-sensitive opsins: cloning, heterologous expression in Drosophila, and physiological characterization. J Neurosci 18:2412-2422.

Warrant EJ, Nilsson DE (1998) Absorption of white light in photoreceptors. Vision Res 38:195-207. 